\title{
The epidemiology of acute kidney injury in critically ill patients in the Gansu Province: the EACG Study
}

\author{
Yu Chen ${ }^{1}$, Fang Feng ${ }^{2}$, Xueni Chang ${ }^{2}$, Dong Liu ${ }^{3}$, Yuan Yuan ${ }^{4}$, Min Li $^{2}$, Jiaojiao Yuan ${ }^{2}$, \\ Chenghua $\mathrm{Mu}^{2}$, and Chenming Dong ${ }^{2}$ \\ ${ }^{1}$ Affiliation not available \\ ${ }^{2}$ Lanzhou University Second Hospital \\ ${ }^{3}$ the 940th Hospital of Joint Logistics Support Force of Chinese People's Liberation Army \\ ${ }^{4}$ People's Hospital of Gansu Province
}

July 7, 2020

\begin{abstract}
Objective: To investigate the epidemiology of acute kidney injury and to clarify the risk factors associated with the prognosis of acute kidney injury in critically ill patients in the Gansu Province.Methods: This was a multicenter, retrospective study. The clinical data of all patients from January 1, 2017, to December 31, 2019, in the intensive care unit of the selected hospitals were screened. Descriptive statistical analysis was performed first, and then the patients were divided into a survival group and a nonsurvival group based on survival status at discharge. RESULTS: (1) Among the 8106 patients admitted, a total of 3019 patients were excluded according to the exclusion criteria. Among the included patients, 890 patients met the diagnostic criteria for AKI, with an incidence of $17.5 \%$ and mortality of $41.3 \%$. Among the selected AKI patients, AKI stage I accounted for $22.02 \%$, AKI stage II accounted for $31.01 \%$, AKI stage III accounted for $46.97 \%$, and $35.8 \%$ of the AKI patients received renal replacement therapy. The higher the AKI stage was, the higher the percentage of renal replacement therapy use. (2) Logistic regression analysis showed that sex, age, AKI stage, RRT, infection, cardio-pulmonary resuscitation, cardiac output, mechanical ventilation, diuretics, white blood cells, platelets, blood urea nitrogen, prothrombin time and activated partial thromboplastin time were significantly associated with the prognosis of acute kidney injury $(\mathrm{P}<0.05)$. Further analysis showed that male sex, old age, high AKI stage, RRT treatment, low cardiac output, mechanical ventilation, elevated leukocyte count, reduced platelet count and reduced serum albumin were independent risk factors for a poor prognosis of AKI. Conclusions: Data from several representative general hospitals in the Gansu Province showed that the incidence and mortality of acute kidney injury in intensive care units were still very high. Sepsis and septic shock are the main causes of AKI.
\end{abstract}

\section{Background}

Acute kidney injury (AKI) is a common critical illness among in-hospital patients, especially critically ill patients.[1-3] Broadly speaking, AKI is a sudden decrease in glomerular filtration rate (GFR) caused by a variety of etiologies and clinical symptoms. Currently, no consensus has been reached on the exact definition of AKI, and more than 30 definitions have been used to diagnose AKI.[4] Due to the different criteria used, the incidence of AKI in critically ill patients has reported to be between $3.2 \%$ and $78 \%$. [5] Moreover, the diversity of AKI definition criteria led to an incomparability among the results of various studies and the inability to accurately evaluate the therapeutic effects of different treatment regimens and interventions for AKI. Although we have made great progress in basic research and the identification and treatment of AKI, the prognosis of AKI is still poor. [6, 7] The incidence and mortality of AKI show significant differences among countries due to the different backgrounds of AKI patients and the diversity of AKI definitions. $[8,9]$ The latest study reported that the incidence of AKI in patients admitted to the Mayo clinic was $6.7 \%$ and that in critically ill patients was $16.9 \%$ between 2006 and 2014[10]. Two other multinational 
prospective studies found that the incidence of AKI was 5.7\% and 57.3\%.[11, 12] However, there are few AKI epidemiological studies among critically ill patients in China, especially in western China, and the data are basically nonexistent. Therefore, this study intends to select three tertiary hospitals in the Gansu Province to obtain epidemiological data of AKI in critically ill patients to infer the epidemiology of AKI in critically ill patients in western China.

\section{Data and methods}

This is a multicenter retrospective observational study about the epidemiology of AKI in ICU patients in Gansu, China. The Ethics Committee of LanZhou University Second Hospital approved this study. The Chinese Clinical Trial Registry number is ChiCTR1800016945. All patients admitted to the ICU in three participating tertiary hospitals in the Gansu Province from January 1, 2017, to December 31, 2019, were retrospectively analyzed.

Inclusion criteria

During the study period (January 1, 2017 to December 31, 2019), all patients admitted to the ICU in three participating tertiary hospitals in the Gansu Province were enrolled.

Exclusion criteria:

1. Chronic kidney disease.

2. History of nephrectomy.

3. Kidney transplantation history within 3 months before ICU admission.

4. Readmission to the ICU during the same hospitalization period.

\section{DESIGN}

The data were recorded in Microsoft Excel, and the research was divided into five steps:

First, all patients admitted to the hospital in 2017 and 2019 were recruited using the Hospital Information System (HIS).

In the second step, the medical records and laboratory examinations were checked one by one for all patients, and patients who met the exclusion criteria were excluded.

The third step was to check the medical records of all AKI patients and extract the data.

The fourth step was to sort out the collected data and import them into Excel.

The fifth step was to import the data into SPSS software for statistical analysis.

\section{Statistics}

SPSS software 21.0 (SPSS Inc. Chicago, IL, USA) was used for statistical analysis. Descriptive statistical analysis was conducted on the enumeration data, and statistical charts were drawn. Then, the characteristics of AKI patients were grouped according to their survival status at discharge. If the measurement data followed a normal distribution, the mean \pm standard deviation $(\mathrm{x} \pm \mathrm{s})$ was used. The t-test was used to compare the two groups, and the q-q normal probability graph was used for the normality test. If the measurement data did not follow a normal distribution, the median $\mathrm{M}$ and quartic interval $(\mathrm{Qu}, \mathrm{QL})$ were used. Comparisons between the two groups were conducted by the Mann-Whitney U test in the Spearman nonparametric test. Enumeration data were expressed as n (\%), Pearson's chi-square test was used to compare binary classification data between the two groups, and the Mann-Whitney U test was used for ordered multiclassification. Logistic regression analysis was used to evaluate the risk factors. First, univariate logistic regression analysis was performed on the demographic data, AKI stage, etiology classification, basic diseases, risk factors, vital signs, laboratory examination results and whether RRT affected the prognosis of AKI patients, and a collinear judgment was made. Then, the variables with statistical significance in the univariate analysis were analyzed by multivariate logistic regression. If the variables were collinear and there were significant differences in the univariate logistic regression analysis, one of the variables was selected 
for multivariate logistic regression analysis. All tests were bilateral, with $\mathrm{P}<0.05$ defined as statistically significant.

\section{Results}

Basic data and general information of the AKI patients

A total of 8,106 patients were admitted to the ICUs of the three hospitals. Of the 3019 patients excluded, the reasons were as follows: 517 patients were $<18$ years old, 1831 patients were admitted to the ICU for $<24$ hours, 482 patients had chronic kidney disease, 16 patients had nephrectomy, 5 patients underwent kidney transplantation within 3 months before admission to the ICU, 52 patients were re-admitted to the ICU during the same hospital stay, and 116 patients had missing needed data for the study (Fig. 1). The characteristics of the AKI patients are shown in Table 1.

Incidence of AKI

According to the KDIGO criteria, a total of 890 ICU patients developed AKI, with an incidence of $17.5 \%$ (890/5087).

AKI stage

According to the KDIGO criteria, AKI can be divided into KDIGO stages (I, II, III), as shown Table 2; this study included $22.02 \%$ (196/890) stage I cases, 31.01\% (276/890) stage II cases, and $46.97 \%$ (418/890) stage III cases.

Etiology of AKI

To clarify the etiology of AKI, the AKI patients were divided into three categories according to etiology: prerenal, renal substantive and postrenal, among which 64\% (570/890) had pre-renal failure. The main causes included 255 cases of sepsis and septic shock, accounting for 44.7\%; 130 cases of reduced cardiac output, accounting for $22.8 \%$; 113 cases of fluid loss from various causes, accounting for $19.8 \%$. A total of $33 \%$ (294/890) had renal substantial renal failure; $16.3 \%$ (48) had renal vascular and glomerular diseases, $75.9 \%$ (223) had renal tubular and interstitial diseases, 7.8\% (23) had other unknown renal injury sites, and 2.7\% (24) had postrenal failure. The main causes were $37.5 \%$ (9) cases of urinary tract stones, $25 \%$ (6) cases of urinary tract tumors, and $29.2 \%$ (9) cases of ureteral strictures, followed by prostate disease and neurogenic bladder.

Continuous renal replacement therapy

Currently, the main treatment methods for AKI include identifying and removing causes and risk factors, treating the primary disease, improving renal insufficiency, providing kidney-preserving drugs, and administering continuous renal replacement therapy (CRRT). CRRT has been an important supportive therapy for patients with AKI, and there are multiple treatment models available based on different mechanisms. RRT procedures were continuous RRT (CRRT). Continuous veno-venous hemofiltration

(CVVH) was the most common mode. Of the 890 AKI patients, 35.8\% (319/890) received CRRT, including $6.3 \%$ (20/319) stage I patients, $21.6 \%$ (69/319 stage II patients), and $72.1 \%$ (230/319) stage III patients. The higher the AKI stage was, the more patients received CRRT.

\section{Outcomes of AKI}

The AKI patients were divided into two groups according to outcome: the survival group and the non-survival group. Of the patients with AKI hospitalized in the ICU, there are 368 deaths during hospitalization, and the mortality rate was $41.3 \%$ overall, $16 \%$ (59) for stage I AKI, $31.5 \%$ (116) for stage II AKI, and $52.4 \%$ (193) for stage III AKI; the higher the AKI stage was, the worse the prognosis. (Table 2) The mortality of patients without RRT was $37.1 \%$ (212), and $48.9 \%$ (156) of patients with RRT died ( $\mathrm{p}=0.001$ ). Further analysis showed that patients treated with RRT had a higher AKI stage and more severe disease than those without RRT. 
Risk factors for death in AKI patients

Univariate analysis

Univariate logistic regression analysis showed that male sex, old age, high AKI stage, RRT, infection, cardiopulmonary resuscitation, reduced cardiac output, mechanical ventilation, diuretics, and reduced white blood cells, platelets, blood urea nitrogen, serum albumin prothrombin time (PT) and activated partial thromboplastin time (APTT) were significantly correlated with death in AKI patients (all $\mathrm{P}$ values were less than 0.05). (Table 3)

Multivariate analysis

Multivariate logistic regression analysis showed that old age, male sex, high AKI stage, reduced cardiac output, mechanical ventilation, RRT, elevated leucocyte count, reduced platelet count, and reduced serum albumin were all independent risk factors for AKI death. (Table 4)

\section{Septic AKI}

Sepsis and septic shock were the most common causes of AKI in critically ill patients. Among the AKI patients enrolled in this study, 28.7\% (255/890) had AKI caused by sepsis and septic shock, which were the major causes of AKI. The main infection sites were the lung, abdomen, urinary system, skin and soft tissue. Among the patients with AKI caused by sepsis and septic shock, 92 patients received RRT, accounting for $28.8 \%$ of the total patients treated with RRT. A total of 136 patients with sepsis and septic shock AKI died, leading to a mortality of $53.3 \%$.

\section{DISCUSSION}

Acute kidney injury is a major clinical problem in critically ill patients that leads to high medical costs and poor prognosis. AKI can arise from a variety of causes and is an important risk factor for chronic kidney disease (CKD). Recently, a southern medical university [13] conducted epidemiological studies on AKI in ICU inpatients in 9 hospitals in different regions of China, but this study did not include the northwest region. Epidemiological investigations have shown that the occurrence and development of diseases will be affected by different regional environments, different study populations, different economic levels and different medical conditions [14-16]. Here, we considered several representative hospitals in the Gansu Province in northwest China as investigation points to conduct a multicenter and retrospective study of 8106 patients admitted to a comprehensive ICU.

The results showed that the incidence of AKI was $17.5 \%$ overall, $22.02 \%$ for stage I, $31.01 \%$ for stage II, and $46.97 \%$ for stage III, and the mortality was $41.3 \%$. A southern medical university study found that, on the basis of the KDIGO diagnostic criteria, the incidence of AKI among ICU inpatients was 30.04\%, and AKI stages I, II, and III accounted for $52.1 \%, 19.7 \%$ and $28.2 \%$ of the patients, respectively. Another regional retrospective study that included five ICU centers in southwest China's Sichuan province reported an incidence of AKI of $37.8 \%$ and hospital mortality as high as 51.8\%.[17] In our study, in comparison, the incidence of AKI was lower, and stage III AKI had for the highest incidence of all stages. The reason may be that AKI lacks specific symptoms and is mainly diagnosed by monitoring the changes in serum creatinine in the short term. A low awareness of AKI would lead to repeated low serum creatinine readings, and the low recognition rate of AKI will cause treatment delays and speed up AKI progression. According to a multicenter survey, the repeated serum creatinine detection rate of hospitalized patients in mainland China was only $29.8 \%$, which is lower than the number reported by developed countries (63.2-67.6\%)[18]. Therefore, it is reasonable to believe that the incidence of AKI in the relatively underdeveloped northwest region may be underestimated.

It is noteworthy that this study found an increased risk of death in male AKI patients compared to in female AKI patients, which was inconsistent with previous studies that showed no significant impact from sex on the prognosis of AKI patients, but a recent study also showed that the male sex was a risk factor for poor 
prognosis in AKI patients[19]. Further studies are needed to confirm the effect of sex on the prognosis of AKI patients.

In addition, in our study, we also found that RRT is one of the risk factors for AKI. Further analyses of the study results showed that RRT in patients with stage III AKI caused most patients to suffer from severe illness, resulting in high mortality. However, according to existing studies, RRT is an important treatment for AKI in ICU patients. Over time, the incidence of AKI patients requiring dialysis increases, but mortality gradually decreases $[20,21]$. This is inconsistent with the results of our study, which may be related to the degree of renal injury, RRT dose, the timing of RRT initiation and other factors that affect the prognosis of AKI patients. There is no unified conclusion at present, and further studies are needed to confirm this hypothesis.

\section{Limitations and Strengths}

1. This study selected three hospitals that can represent the medical situation of the Gansu Province. From the perspective of epidemiological sampling, there is sampling bias, which may affect the epidemiological results for AKI. In the future, more data from hospitals of different regions and levels can be collected for further research.

2. Our study is a retrospective study and thus cannot accurately monitor the urine volume of patients, which may underestimate the incidence of acute kidney injury.

Although this study has some shortcomings, it is also innovative and practical, which is of great benefit for guiding clinical treatment. First, epidemiological studies on acute kidney injury in critically ill patients are mostly single-center, small-sample studies with poor representativeness. Our study is a multicenter study with a large sample size, which is closer to the overall sample size. The results of our study are more conducive to guiding clinical treatment and scientific research. Second, laboratory indicators were added to the analysis of prognostic risk factors in our study, and these indicators were simple and economical to obtain and had good clinical application prospects. At present, most scholars are committed to studying the pathogenesis of AKI and the risk factors affecting the occurrence of AKI, while research on the factors affecting the prognosis of acute kidney injury has relatively lagged behind. In the future, predicting the prognosis of AKI will arouse the interest of many researchers. Most importantly, with the rapid development of big data in medicine, an increasing number of people will realize the importance of data science. The development of medical science and technology is also inseparable from big data. Specialized development, collation and analysis of big data may bring unexpected benefits to our scientific research. Therefore, our research "The epidemiology of acute kidney injury in critically ill patients in the Gansu Province (EACG)" series (Chinese clinical trial registration number: ChiCTR1800016945) will further expand upon the data collected from critically ill patients with AKI in the Gansu Province, establish a database and build a crossfield data sharing platform to help more clinical and scientific researchers interested in AKI quickly obtain clinical data and process these data. Furthermore, data from China, the world's largest developing country, will provide valuable information for improving the current situation and economic burden of acute renal impairment worldwide.

Conclusion

Large-scale epidemiological data from several representative general hospitals in the Gansu Province showed that the incidence and mortality of acute kidney injury in intensive care units were still very high. Sepsis and septic shock are the main causes of AKI.

\section{Declarations}

\section{Ethics approval and consent to participate}

The Ethics Committee of LanZhou University Second Hospital approved this study, and the number was 2018-043. Written informed consent was obtained from individual or guardian participants.

\section{Consent for publication}


Not applicable.

\section{Availability of data and materials}

All data generated or analysed during this study are included in this published article.

\section{Competing interests}

All authors declare that there are no competing interests.

\section{Funding}

Not applicable.

\section{Authors' contributions}

Conducted the study: YC, FF.

Collected all data: DL, YY, ML, J-jY, C-hM, C-mD.

Did the statistical analysis: YC, X-nC.

\section{Acknowledgements}

We would like to thank AJE (www.aje.cn) for English language editing.

\section{Authors' information}

${ }^{1}$ Intensive Care Unit 2 of Lanzhou University Second Hospital;

${ }^{2}$ Intensive Care Unit of the 940th Hospital of Joint Logistics Support Force of Chinese People's Liberation Army;

${ }^{3}$ Intensive Care Unit of People's Hospital of Gansu Province;

${ }^{4}$ Nursing Department of Lanzhou University Second Hospital;

[1] Mehta RL,Kellum JA,Shah SV, et al. Acute Kidney Injury Network: report of an initiative to improve outcomes in acute kidney injury. Critical Care[J]. 2007;2(11).

[2] Cheng X,Wu B,Liu Y, et al. Incidence and diagnosis of Acute kidney injury in hospitalized adult patients: a retrospective observational study in a tertiary teaching Hospital in Southeast China. Bmc Nephrology[J]. $2017 ; 1(18)$.

[3] Khwaja,Arif. KDIGO Clinical Practice Guidelines for Acute Kidney Injury. Nephron Clinical Practice[J]. 2017;4(120):179-84.

[4] Kellum JA,Levin N,Bouman C, et al. Developing a consensus classification system for acute renal failure. Current Opinion in Critical Care[J]. 2002;6(8):509-14.

[5] Case J,Khan S,Khalid R, et al. Epidemiology of Acute Kidney Injury in the Intensive Care Unit. Crit Care Res Pract[J]. 2013(2013):1-9.

[6] Clec'H C,Gonzalez F,Lautrette... A. Multiple-center evaluation of mortality associated with acute kidney injury in critically ill patients: a competing risks analysis. Critical Care[J]. 2011;3(15).

[7] Vaara ST,Pettila V,Reinikainen M, et al. Population-based incidence, mortality and quality of life in critically ill patients treated with renal replacement therapy: a nationwide retrospective cohort study in Finnish intensive care units. Critical care (London, England)[J]. 2012;1(16):R13.

[8] Susantitaphong P,Cruz DN,Cerda J, et al. World Incidence of AKI: A Meta-Analysis. Clinical Journal of the American Society of Nephrology Cjasn[J]. 2013;9(8):1482-93. 
[9] Hoste EAJ BS, Bellomo R, Cely CM, Colman R, Cruz DN, . Epidemiology of acute kidney injury in critically ill patients: the multinational AKI-EPI study. Intensive care medicine[J]. 2013;8(41):1411-23.

[10] Kashani K,Shao M,Li G, et al. No increase in the incidence of acute kidney injury in a population-based annual temporal trends epidemiology study. Kidney International[J]. 2017:S0085253817302156.

[11] Uchino S,Kellum JA,Bellomo R, et al. Acute Renal Failure in Critically Ill Patients: A Multinational, Multicenter Study. Jama the Journal of the American Medical Association[J]. 2005;7(294):813-8.

[12] Bagshaw SM,George C,Bellomo R. Changes in the incidence and outcome for early acute kidney injury in a cohort of Australian intensive care units. Critical Care[J]. 2007;3(11).

[13] LEI Y NS, SUN DH. Epidemiology of acute kidney injury in Chinese critical patient. J South Med Univ,2016(in Chinese)[J]. 2012;6(36):744-50.

[14] Uchino S,Bellomo R,Bagshaw SM, et al. Transient azotaemia is associated with a high risk of death in hospitalized patients. Nephrol Dial Transplant[J]. 2010;6(25):1833-9.

[15] Mehta RL,Cerda J,Burdmann EA, et al. International Society of Nephrology \"s 0by25 initiative for acute kidney injury (zero preventable deaths by 2025): a human rights case for nephrology. Lancet[J]. 2015;9987(385):2616-43.

[16] Yang L,Xing G,Wang L, et al. Acute kidney injury in China: a cross-sectional survey. Lancet[J]. 2015;10002(386):1465-71.

[17] Zhou J,Liu Y,Tang Y, et al. A comparison of RIFLE, AKIN, KDIGO, and Cys-C criteria for the definition of acute kidney injury in critically ill patients. International Urology \& Nephrology[J]. 2015;1(48):125-32.

[18] Xu X,Nie S,Liu Z, et al. Epidemiology and Clinical Correlates of AKI in Chinese Hospitalized Adults. Clinical Journal of the American Society of Nephrology Cjasn[J]. 2015:CJN.02140215.

[19] Sawhney S,Beaulieu M,Black C, et al. Predicting kidney failure risk after acute kidney injury among people receiving nephrology clinic care. Nephrol Dial Transplant[J]. 2018.

[20] Wald R,McArthur E,Adhikari NK, et al. Changing incidence and outcomes following dialysis-requiring acute kidney injury among critically ill adults: a population-based cohort study. American journal of kidney diseases : the official journal of the National Kidney Foundation[J]. 2015;6(65):870-7.

[21] Lai CF,Wu VC,Huang TM, et al. Kidney function decline after a non-dialysis-requiring acute kidney injury is associated with higher long-term mortality in critically ill survivors. Critical care (London, England)[J]. 2012;4(16):R123.

\section{Hosted file}

Table 1. docx available at https://authorea.com/users/339782/articles/465935-the-epidemiologyof-acute-kidney-injury-in-critically-ill-patients-in-the-gansu-province-the-eacg-study

\section{Hosted file}

Table 2.docx available at https://authorea.com/users/339782/articles/465935-the-epidemiologyof-acute-kidney-injury-in-critically-ill-patients-in-the-gansu-province-the-eacg-study

\section{Hosted file}

Table 3.docx available at https://authorea.com/users/339782/articles/465935-the-epidemiologyof-acute-kidney-injury-in-critically-ill-patients-in-the-gansu-province-the-eacg-study

\section{Hosted file}

Table 4.docx available at https://authorea.com/users/339782/articles/465935-the-epidemiologyof-acute-kidney-injury-in-critically-ill-patients-in-the-gansu-province-the-eacg-study 


\section{Hosted file}

Fig.1.docx available at https://authorea.com/users/339782/articles/465935-the-epidemiologyof-acute-kidney-injury-in-critically-ill-patients-in-the-gansu-province-the-eacg-study 